Rev. SINAPSIS, Edición № 11, Vol. 2, Diciembre 2017

ISSN $1390-9770$

\title{
Eficacia y seguridad del cloruro de sodio $7.5 \%$ - Hemohest en el tratamiento inicial del choque hemorrágico traumático.
}

Efficacy and safety of sodium chloride $7.5 \%$ - Hemohest in the initial treatment of traumatic hemorrhagic shock.

Secundino González Pardo.Dr, MSc ${ }^{(1)}$

Itala Silvana Paspuel Yar .MSc ${ }^{(2)}$

Arlines Piña-Tornés. Dra, MSc ${ }^{(3)}$

(1) Universidad Técnica del Norte, Facultad Ciencia de la Salud, Ibarra- Ecuador, sgonzalez@utn.edu.ec

(2) Universidad Técnica del Norte, Facultad Ciencia de la Salud, Ibarra- Ecuador, papuelsilvana@gmail.com

(3) Universidad de Guayaquil, Facultad de Odontología, Guayaquil-Ecuador, arlines.pinat@ug.edu.ec Contacto: sgonzalez@unt.edu.ec

Receptado: 15/09/2017

Aceptado: 10/11/2017

\section{Resumen:}

Se realizó un ensayo clínico fase II explorativo, aleatorizado y controlado, en la sala de emergencias del hospital provincial universitario "Carlos Manuel de Céspedes", Bayamo, provincia Granma, en el período comprendido entre Septiembre 2013 a Septiembre de 2016 con el objetivo evaluar la eficiencia y seguridad del $\mathrm{ClNa}$ 7.5\% Hemoest en el tratamiento del choque hemorrágico. El universo construido por los pacientes politraumatizados a los que se le diagnostique estado de choque hemorrágico traumático clase II y III. Se incluyeron 64 pacientes de 15 a 60 años de edad. Ambos tratamientos se aplicaron de forma intravenosa, a dosis según requerimiento del paciente. Se aplicaron las Normas Nacionales e Internacionales de Buenas Prácticas Clínicas. Para determinar la eficacia se realizó la prueba unilateral de equivalencia de las proporciones a la variable principal del estudio: (respuesta clínica). La seguridad del tratamiento fue evaluada a través del tipo e intensidad del evento adverso y su relación de causalidad con el tratamiento recibido. Los grupos resultaron ser homogéneos en cuanto a las características de base estudiadas, las que no mostraron influencia en la respuesta al tratamiento. El análisis con los pacientes que completaron el período de tratamiento con $\mathrm{ClNa}$ 7.5\% Hemoest tuvieron una evolución satisfactoria 
Rev. SINAPSIS, Edición № 11, Vol. 2, Diciembre 2017

ISSN 1390 - 9770

y se concluyó que es más efectivo y seguro a las dosis según el esquema de administración aplicados en el estudio que el tratamiento utilizado en como control.

Palabras clave: Solución Salina CLNA 7.5\%-Hemest, Schok Hemorrágico

\begin{abstract}
.
It has been made a clinic essay phase II explorative, randomized and controlled, in the emergency room of the University province hospital "Carlos Manuel de Céspedes", Bayamo, Granma province, in the period between September 2013 to September 2016 with the objective of test the efficiency and security of CINa $7.5 \%$ Hemoest in the treatment of hemorrhage shock. The universe constructed by patients polytraumatized, who were diagnosed state of hemorrhagic shock clase I and II. 64 patients were include, with ages between 15-60 years. Both treatments was applied in intravenous form in doses according to the requirements of the patient. National and International norms of Good Medical Practices was applied. To determine the efficiency it was made the unilateral test of equivalence of the proportions to the main variable of the study :( Clinic response). The security of the treatment was tested through the type and intensity of the adverse event and its causal relationship with the treatment received. The groups turned out to be homogeneous in terms of the characteristics of bases studied, which showed no influence on the response to the treatment. Analysis with patients who completed the treatment period with $\mathrm{CINa} 7.5 \%$ Hemoest had a satisfactory evolution and it is concluded that it is more effective and safe at the doses according to the administration scheme applied in the study, than the treatment used as control
\end{abstract}

Key words: Saline solution CLNA 7.5\%-Hemoest, hemorrhage shock.

\title{
Introducción:
}

El choque es un síndrome clínico, que consiste en la súbita disrupción de la función circulatoria e insuficiencia general de la perfusión tisular la cual últimamente conduce a alteración de los mecanismos homeostáticos. En general, todos los estados de choque eventualmente envuelven entrega disminuida o falla en la utilización de substratos celulares esenciales, los cuales causan disfunción, eventual pérdida del metabolismo y función celular (Cecconi, De Backer, Antonelli, \& Beale, 2014), (Bouglé, Harrois, \& Duranteau, 2012).

Durante el choque hemorrágico descompensado, con una pérdida aproximadamente del 20-40\% del volumen intravascular; los mecanismos compensadores que restablecen la perfusión tisular son rebasados y hay disminución en la tensión arterial media (TAM), taquicardia, oliguria, hipoperfusión de 
órganos vitales, choque irreversible, disfunción orgánica múltiple (DOM) y muerte del paciente, sobre todo si la hemorragia no es controlada (Burton, Britton, \& Todd, 2014), (Sigüenza \& Vicuña, 2015).

Luego de diagnosticar y clasificar la pérdida sanguínea, es importante tratar de establecer la etiología del choque. El trauma es la principal causa de choque hemorrágico y se asocia a una elevada mortalidad, ocurre en la mayoría de los casos en menores de 44 años, constituye la primera causa de muerte en este grupo y es a su vez la tercera causa de muerte en general (Bouglé, Harrois, \& Duranteau, 2012).

Según la Organización Mundial de la Salud (OMS), cada año mueren en el mundo 5,5 millones de personas como consecuencia de lesiones causadas por violencia accidental o intencionada y son responsables del 16\% de la carga global de enfermedad. Muchas de estas muertes son prevenibles. En los Estados Unidos es la primera causa de muerte en menores de 45 años y la cuarta en todos los grupos de edad.

El costo anual generado por las lesiones traumáticas alcanza un promedio de 118.000 millones de dólares, números crecientes de muertes previsibles y de pacientes inhabilitados. En Colombia constituye uno de los principales problemas de salud pública, por sus implicaciones sociales, económicas y morales. Según datos publicados por el Departamento Administrativo Nacional de Estadística de este país, las muertes violentas ocupan el primer lugar entre las causas de mortalidad, por encima del cáncer y de las enfermedades cardiovasculares.

El grupo de población más afectado se encuentra entre los 15 y los 45 años, promedio de 23 años; con resultados económicos nefastos por la pérdida de años de vida productiva. Además de estas consecuencias ponderables, existen otras de carácter familiar y social verdaderamente inconmensurables (Gómez, Ayuso, \& Jiménez, 2008), (Navarro, Koo, Orrego, \& Muñoz, 2014).

En los últimos años los cambios en el perfil epidemiológico en Cuba han colocado a los accidentes dentro de los primeros lugares de la mortalidad, y es la cuarta causa de defunción general, con 4890 defunciones en 2012 para una tasa de mortalidad de 44 por 100000 habitantes. Entre los accidentes, los de tránsito se destacan sobre los demás, a tal extremo que durante los años de la década de los 90 alcanzó la cifra de 26362 muertos en nuestro país, o sea, que se produjo una muerte cada 4 horas por este motivo (Egea, Freire, \& Serrano, 2014). Como se puede apreciar es una enfermedad muy frecuente con alta mortalidad, por lo que la actuación médica adecuada es importante. 
Uno de los problemas a los que enfrenta a diario el médico en la atención al trauma es la reposición de fluidos en el paciente con choque hemorrágico, por su repercusión en todos los sistema y órganos, privando a los tejidos de oxígeno y nutriente suficientes y la consiguiente disminución del rendimiento cardiaco (Scalea, Maltz, \& Yelon, 2014) (Shoemaker, Appel, \& Kram, 1999), (Scheeren, Wiesenack, Gerlach, \& Gernot, 2013).

En los últimos años en base a nuevos modelos experimentales de hemorragia y a la experiencia clínica adquirida en cirugía de trauma, anestesiología y medicina intensiva se abre un nuevo campo de investigación que rompe el paradigma de la reanimación convencional, para dar inicio a nuevas alternativas terapéuticas en el manejo del choque hemorrágico como son: la hipotensión permisiva o reanimación con hipotensión y la reanimación retardada. Como regla general muchos protocolos de tratamientos han generalizado el tratamiento con soluciones a base de cristaloides y colindes, sin embargo, en este punto no existe un acuerdo al respecto (Pinsky, 2014), (Raghunathan, y otros, 2014), (Shoemaker, Appel, \& Kram, Hemodynamic and oxygen transport responses in survivors and nonsurvivors of high risk surgery, 1993).

Todos los elementos hasta ahora expuesto señalan la necesidad de un mayor conocimiento sobre la problemática en cuestión. Como objeto de la investigación se considera a los pacientes con choque hemorrágico traumático.

Con estos antecedentes y porque aún el reemplazo de volumen tiene el objetivo de lograr una adecuada perfusión al nivel celular y evitar que se sobreañada al proceso de isquemia el de reperfusión y no existe un fármaco eficaz para el tratamiento del choque hemorrágico, la presente investigación dio respuesta al problema científico: ¿Resulta eficaz y seguro el empleo de las soluciones de cloruro de sodio 7,5\%Hemohest como opción terapéutica en el choque hemorrágico?

Como objetivo general se evaluó la eficacia y la seguridad del cloruro de sodio al 7,5\% - Hemohest en el tratamiento del choque hemorrágico traumático.

Se partió de la hipótesis de que el tratamiento con cloruro de sodio al $7.5 \%$ - Hemohest es considerada equivalente a la terapia con cloruro de sodio al $0.9 \%$ si la diferencia entre las proporciones de pacientes en los que muestra efectividad no es mayor de un $20 \%$ entre ambos grupos, siendo menor la proporción del cloruro de sodio al $7.5 \%$ - Hemohest. El porciento de eficacia esperada para el cloruro de sodio al 0.9 es de un $90 \%$. 
La evaluación clínica del enfermo con choque hemorrágico traumático fue importante la experiencia del intensivista, se consideró que, en el orden práctico este estudio permite la correcta utilización de la resucitación con fluidos en pacientes con esta entidad, lo cual sería muy útil para brindar una mejor atención médica a estos enfermos, lo cual traduciría menor mortalidad.

El conocimiento de esta opción terapéutica es realmente conveniente si se tiene presente el aumento de este proceso en Cuba y en Granma. Por otra parte, la no existencia de estudio donde se evalúen la eficacia y seguridad de la sustancia objeto de investigación en la mencionada enfermedad en nuestro entorno, resulto necesario la realización de investigaciones clínicas sobre factores objetivos, fáciles de aplicar y sin necesidad de grandes recursos materiales y técnicas engorrosas.

Desde el punto de vista social esta investigación es válida debido a que el choque hemorrágico traumático es una enfermedad frecuente, creciente y elevada mortalidad, en países tanto desarrollados como en vía de desarrollo, lo que hace necesario la realización de estudios encaminados a mejor la atención de estos enfermos y así garantizar mayor calidad de vida.

Su aporte teórico está dado en probar la eficacia y seguridad del cloruro de sodio al $7.5 \%$ y Hemohest, debido a que los conocimientos al respecto son ambiguos. En este trabajo se utilizó para su diseño y análisis estadístico los recursos que brinda la Epidemiología Clínica para los ensayos clínicos e interpreta sus resultados basado en los principios fisiopatológicos y conceptos epidemiológicos, valorando su utilidad y aplicabilidad.

\section{Materiales y métodos:}

Se realizó un ensayo clínico fase II Explorativo, aleatorizado, controlado, con el objetivo evaluar la eficiencia y seguridad del ClNa 7.5\% Hemoest en el tratamiento del choque hemorrágico en la sala de emergencias del Hospital Provincial Universitario "Carlos Manuel de Céspedes", Bayamo, Provincia Granma, ingresados en el período comprendido entre septiembre 2013 a septiembre de 2016. Se aplicaron las Normas Nacionales e Internacionales de Buenas Prácticas Clínicas.

El universo construido por los pacientes politraumatizados a los que se le diagnosticó un estado de choque hemorrágico clase II y III. Quedando el estudio constituido por 64 pacientes que se asignaran aleatoriamente en de dos grupos de 32 pacientes. 
Rev. SINAPSIS, Edición № 11, Vol. 2, Diciembre 2017

Criterios de inclusión: Diagnóstico de choque hemorrágico clase II y III, de ambos sexos de las edades comprendidas entre 16 y 60 años, sin terapia previa al de su ingreso, voluntariedad del paciente para participar en el estudio.

Criterios de exclusión: Pacientes embarazadas, pacientes con trastornos psiquiátricos, pacientes que presente antecedentes de insuficiencia renal, enfermedades cardiovasculares, pacientes sin pulso ni actividad eléctrica al momento del ingreso, pacientes quemados con manifestaciones de choque hipovolémico.

El estudio se realizó en correspondencia con lo establecido en la Declaración de Helsinki, versión correspondiente a la 52a Asamblea General de Edimburgo, Escocia, octubre del 2000. Además, rigió por las regulaciones estatales vigentes en la República de Cuba. Se informó a los pacientes o acompañantes legales las características del estudio, y estos confirmaron voluntariamente, por escrito, su disposición para participe en el ensayo mediante el Modelo de Consentimiento Informado; el cual será firmado y fechado por cada uno de ellos y por el Investigador Responsable (IR) de cada institución participante en la investigación.

Tratamiento: Se conformaron dos grupos denominados Grupo A (Control) y Grupo B (Estudio), según orden de llegada. Los pacientes que correspondan a números pares formarán parte del Grupo A y recibirán el protocolo de tratamiento A. Los pacientes pertenecientes a números impares al grupo B y recibirán el protocolo de tratamiento $\mathrm{B}$. Se estableció un protocolo para la inclusión y tratamiento de los pacientes portadores de choque hemorrágico que cumplan con los criterios de selección, el cual fue aplicado por el médico de la sala de emergencia. Este protocolo contará con dos partes:

Protocolo de tratamiento específico para cada grupo: Tratamiento A: Solución de CINa 0.9\% a razón de $55 \mathrm{ml} / \mathrm{Kg}$. de peso corporal para 24 horas, en bolos de $250 \mathrm{ml}$ - $300 \mathrm{ml}$. Tratamiento B: ClNa 7.5\% más hemoest a razón de $15 \mathrm{ml} / \mathrm{Kg}$. de peso para las 24 horas. Se iniciará con la administración de $250 \mathrm{ml}$, de ellos el $6 \%$ de hemoest. Antes de aplicar los diferentes protocolos de tratamientos se registrarán y anotarán los parámetros tensión arterial sistólica(TAS), tensión arterial diastólica(TAD), tensión arterial media(TAM), frecuencia cardiaca(FC), saturaciones de oxihemoglobina ( $\mathrm{HbO} 2)$, saturación parcial de oxigeno(SPO2) frecuencia respiratoria(FR), magnitud del trauma (según escala de trauma), Nivel de conciencia (escala de Glasgow) y diuresis, según los métodos tradicionales. Se hizo control de la respuesta al tratamiento cada 30 minutos y las dos primeras horas, se evaluó de la efectividad del tratamiento a la hora, a las dos horas, 24 horas y al alta del paciente por el investigador. 
Rev. SINAPSIS, Edición № 11, Vol. 2, Diciembre 2017

ISSN $1390-9770$

Las variables empleadas para medir el efecto y seguridad; fueron variables principales de respuesta (tensión arterial media, diuresis, saturación de oxígeno); las variables secundarias de respuesta (frecuencia cardiaca, frecuencia respiratoria, nivel de conciencia, llene capilar) y las variables de control (edad, sexo). La efectividad del tratamiento se evaluó estableciendo tres categorías (mejorado, igual y empeorado, todos con respecto a la primera hora).

La seguridad del producto de evaluó a través de los eventos adversos que pudieron ocurrir durante la administración de los tratamientos, el investigador efectuó el interrogatorio y examen físico al paciente buscando los posibles signos o síntomas que evidenciaran la presencia de reacciones o eventos. Esto se realizó en las consultas evolutivas efectuadas a los 60, 120 minutos, 24 horas y al alta de administrados los tratamientos.

Los eventos adversos fueron clasificados de acuerdo a su intensidad como ligero, moderado, severo, grave y eventos adversos inesperados. Las técnicas estadísticas que se aplicaron para estudiar el comportamiento de las variables involucradas en el ensayo y los test utilizados fueron, para el análisis de homogeneidad en el caso de edad se aplicó la prueba de diferencia de media para muestra no relacionada, referente a la etiología y la localización anatómico se utilizó el test de Chi-cuadrado; se trabajó con un nivel de confianza del 95\%.

Para comprobar la normalidad de las variables de estudio (TAM, $\mathrm{SPO}_{2}$ y diuresis) en cada uno de los momentos evaluados, se utilizó la prueba de Kolmogorov - Smirnova por tratarse de una muestra superior a 50 individuos con un nivel de significación de 0.05.Como hipótesis nula $\left(\mathrm{H}_{0}\right)$ se asumió en cada uno de los momentos que las variables en cuestión se distribuyen normalmente. Como hipótesis alternativa $\left(\mathrm{H}_{1}\right)$ se asumió que la variable objeto de estudio no se distribuye normalmente.

Se consideró significancia estadística para valores de probabilidad inferior a 0.05. Para el caso que la variable no se distribuyera normalmente se aplicó la prueba no paramétrica Mann- Whitneg realizándose un análisis vertical y se definieron las variables estadísticas de la siguiente forma; hipótesis nula, no hay diferencias significativas entre ambos grupos, hipótesis alternativa, hay diferencias entre ambos grupos.

\section{Resultados.}

Se evaluaron en el ensayo un total de 104 pacientes entre 15 y 60 años de edad, resultando no incluidos 36, por no cumplir con los criterios de inclusión para un 34,6\%. Se incluyeron y se aleatorizaron 62 
pacientes, 32 fueron asignados al grupo del Cloruro de sodio al 7.5\%-hemoest y 32 al grupo con Cloruro de sodio al $0.9 \%$. No hubo interrupción del tratamiento.

En la tabla (1) se observo que tipo de localización anatómica de la lesión predominó a nivel del abdomen en los ambos grupos, en el experimental con 15 pacientes $(46,9 \%)$ y en los controles 17 casos $(53,1 \%)$. No hubo diferencias significativas entre ambos grupos. La etiología más frecuente fue el arma blanca en el grupo experimental con 14 pacientes $(43,7 \%)$ y en los controles fue los accidentes de tránsitos 17 casos $(40,6 \%)$. No se encontró diferencias significativas.

En la tabla (2) se observa el comportamiento de normalidad de la tensión arterial media en los distintos momentos de su medición donde existen diferencias significativas de 0,010 de la TAM pre tratamiento y de los demás momentos; para el comportamiento de la frecuencia cardiaca se procedió de igual manera aplicando la prueba de Mann- Witnev. Se puede observar que este parámetro tuvo un descenso progresivo desde una media de 125,56 LPM (Latido por minutos) en el grupo experimental y 132,3 LPM en el grupo control con una probabilidad de 0, 031 hasta 104, 43 LPM y 110, 15 LPM en los grupos experimental y control respectivamente, con una probabilidad de 0,000, lo que muestra diferencias significativas entre los valores de la frecuencia cardiaca entre el grupo estudio y el control.

Al analizar Comportamiento de la TAM en ambos grupos al inicio del tratamiento para diferencia de medias para muestras independientes en la tabla (3) se observa que la media del grupo experimental se sitúa en 49, 33 con una desviación típica de 11,60 y en el grupo control la media en 51,43 con una desviación típica de 12,48 para una probabilidad de 0,48, lo que muestra ausencia de diferencias significativas.

Para determinar la efectividad del tratamiento con CINa 7.5\% y del Hemohest en el choque hemorrágico según los cambios ocurridos con la TAM en los diferentes momentos se puede observar que este parámetro tuvo un ascenso progresivo desde una media de 49.3mm $\mathrm{Hg}$ con una desviación típica de 11,60 en el grupo experimental y de 51,4 mm Hg con una desviación típica de 12,48 en el grupo control con una probabilidad de 0,48 antes del tratamiento como muestra la tabla (3) hasta 83,06 en el grupo experimental y 83,22 en el grupo control, no existen diferencias significativas entre ambos grupos. .

Para investigar el comportamiento de la saturación de oxígeno se hizo de forma análoga al método seguido para la TAM y frecuencia cardiaca, como se muestra en las tablas (4 y 5) no fue normal, por lo que se le aplicó la prueba de Mann- Witnev. 
Rev. SINAPSIS, Edición № 11, Vol. 2, Diciembre 2017

ISSN 1390 - 9770

Se puede observar que este parámetro tuvo un ascenso progresivo desde las mensuraciones realizadas pre tratamiento hasta el alta con una media desde 87,47 \% en el grupo experimental y 91,90 \% LPM en el grupo control con una probabilidad de 0, 000 hasta 97,94 \% y 92,56\% con una probabilidad de 0,000 en los grupos experimental y control respectivamente.

Como se muestra en la tabla ( 4 y 6 ) el comportamiento de la variable diuresis pre tratamiento no fue normal, por lo que se le aplico la prueba de Mann- Witnev. No hubo diferencias significativas pretratamiento entre ambos grupos.

Para las mediciones hechas en los momentos después del tratamiento en los que el comportamiento fue normal, tabla (3) se aplicó el estadístico W de Wilcoxon, observandose diferencias significativas entre el grupo experimental y los controles a las 24 horas de tratamiento.

Durante el estudio se presentaron eventos adversos en 5 pacientes que recibieron tratamiento con Clna $7,5 \%$ y Hemohes, lo que representa un $15,6 \%$ de los pacientes de este grupo y en el grupo control se presentaron 7 para $21,9 \%$. Hubo diferencias estadísticamente significativas con respecto a esta variable de estudio $(\mathrm{p}=0.029)$ entre ambos grupos.

\section{Discusión.}

Los medicamentos y sus usos son tan viejos como la humanidad, con más de 4000 años de uso, pero sus regulaciones ocurrieron a partir de la conferencia de Helsinki, en junio de 1964. A partir de ahí estas directrices se han ido fortaleciendo.

Estos hechos han proporcionado que el uso en humanos este bien establecidos en cuanto a dosis, duración del tratamiento y efectos adversos. Es precisamente en la urgencia y la emergencia donde juega un papel de extraordinaria importancia este conocimiento.

En el año 2010, los accidentes y las heridas por arma blanca constituyeron la cuarta causa de muerte con una tasa bruta de 39,1 por 100000 habitantes, lo que representó un promedio de 7,2 años de vida potencialmente perdidos. Por grupos de edad son la primera causa de muerte de 1 a 4 y de 10 a 19 años; con una tasa de 1.1 y 12.6 por 100000 habitantes respectivamente. ${ }^{5}$ Lo que coincide con la presente investigación.

Las lesiones que habitualmente producen choque son en orden de frecuencia el tórax y el abdomen seguido de los traumas craneales, según varios autores (Shoemaker, Appel, \& Kram, Hemodynamic and 
Rev. SINAPSIS, Edición № 11, Vol. 2, Diciembre 2017

oxygen transport responses in survivors and nonsurvivors of high risk surgery, 1993), (Serracant, y otros, 2016). lo que coincide con la presente investigación. Como explicación tendríamos que en estos sitios anatómicos existe mayor número de estructuras vascularizadas y órganos vitales que sus lesiones provocaría pérdida importante de sangre (Serracant, y otros, 2016).

Una mejor respuesta de la tensión arterial media en pacientes traumatizados tratados con solución Cloruro de Sodio al 7.5 \% en comparación con los tratados solución Ringer lactato (34 +/- 46 vs 11 +/$49 \mathrm{~mm} \mathrm{Hg}, \mathrm{P}<.03)$ fue descrito por Basar MJ y colaboradores del departamento de cirugía de la universidad de California los que encontraron un incremento significativo en la presión arterial sistólica al arribo al departamento de emergencia de pacientes tratados con solución salina (Kapp, 2005). Estos resultados coinciden con los del presente trabajo.

En un estudio aleatorizado, controlado en el que utilizó soluciones de hipertónicas e hiperoncóticas para mejorar la función cardiaca en los niños después de la cirugía a corazón abierto, se encontró que el índice cardíaco era 3.6 + / - 0.26 L/min por el $\mathrm{m}^{2}$ antes de la administración de volumen y aumentó a 5.96 + / 0.27 después de la administración de la solución. Este estudio mostró un aumento profundo de índice cardíaco posterior de la aplicación de solución del hipertónica-hiperoncótica en los niños después de la cirugía a corazón abierto sencilla, haciendo pensar en un efecto inotrópico positivo. El importe global de catecolamina era más bajo, mientras asumiendo esa solución hipertónica-hiperoncótica reduce la necesidad por el apoyo del inotrópico positivo. El efecto cardíaco positivo observado de la solución hipertónica-hiperoncótica incluso puede ser intensificado por una precarga disminuida (disminuyó el índice de resistencia vascular sistémico). Según la relación de Frank-estornino, una herramienta eficaz en el tratamiento de rendimiento cardíaco bajo, es una precarga elevada mientras la post carga se disminuye (Rocha \& Poli de Figueiredo, 2014), (Chang, John, \& Holcomb, 2017), (Schreiber, y otros, 2015).

La frecuencia cardiaca es también un factor principal para la función miocárdica. Las interacciones de la frecuencia cardiaca contribuyen al gasto cardíaco, la contractilidad y la interrelación aporte-demanda de oxígeno miocárdico. Se reconoce como un factor mayor en la determinación del gasto cardíaco, como el producto de volumen latido y frecuencia cardiaca, y también se reconoce que una taquicardia excesiva puede disminuir el volumen latido, esto reduciendo el tiempo del llenado ventricular. Aumentando la frecuencia cardiaca, incremento del consumo de oxígeno miocárdico y disminuye el tiempo de perfusión 
Rev. SINAPSIS, Edición № 11, Vol. 2, Diciembre 2017

ISSN $1390-9770$

coronaria (diástole) con lo cual se altera el aporte- demanda de oxígeno por el miocardio (Jamal, Mandel, \& Barnea, 2014).

Aunque los signos clínicos en el choque reflejan mal los cambios en la volemia y generalmente son tardíos, como se explicaba con anterioridad en su evolución si es importante monitorizar variables tales como frecuencia cardiaca y saturación arterial de oxígeno. En este estudio estas variables muestran un mismo patrón, dado por mejoría, lo que corrobora la eficacia del tratamiento experimental, superior al grupo control (Rossaint, Bouillon, Cerny, \& Coats, 2016).

El choque actualmente dejó de ser visto como un evento simple, determinado por disminución en presión arterial, gasto cardiaco y diuresis; más que eso el choque plantea un problema complejo donde hay falta de acoplamiento entre la disponibilidad y el consumo de oxígeno, entonces los esfuerzos deben ir dirigidos más allá del solo hecho de elevar la presión arterial, el objetivo principal del manejo debe ser mejorar la perfusión tisular la cual puede ser monitorizada de forma indirecta por la respuesta al tratamiento de las variables antes expuestas (Rossaint, Bouillon, Cerny, \& Coats, 2016), (Guerrero \& Andrighetti, 2011).

A nivel renal se reduce la perfusión glomerular y la formación de orina. Sin embargo, esta redistribución del flujo sanguíneo circulante supone hipoxia e hipoperfusión oculta en territorios sistémicos que no siendo vitales a corto plazo (territorio esplácnico), tienen importancia capital en la morbilidad y mortalidad a mediano y largo plazo en los pacientes en estado de choque. Los hallazgos encontrados en la presente investigación serían explicables por los mecanismos farmacológicos de ambas sustancias.

La solución salina al $0.9 \%$ tienen un corto periodo de permanencia intravascular y se acumulan en el espacio intersticial por lo que se requieren dosis de al menos tres veces la estimación de la pérdida volémica. No producen efectos de hipersensibilidad y carecen de dosis límite para su infusión intravascular, aunque debe monitorizarse el grado de edematización derivados de su uso y el riesgo de acidosis hiperclorémica secundario al uso masivo de solución fisiológica. Sus efectos sobre la hemostasia son mínimos a condición de que existan niveles de hemodilución inferiores al 50\% del volumen sanguíneo (Rodríguez, Echevarría, \& Díaz, 2015), (Perel, Roberts, \& Ker, 2013).

Mientras que la solución salina hipertónica (7.5\%) asociada o no a coloide tiene un rápido y elevado poder de relleno vascular por deshidratación intersticial e intracelular. Algunas de las preparaciones que están empezando a ser usadas son: Solución salina al $7.5 \%$ más dextrano 70 al 6\%, o la Hiper- 
Hidroxietilalmidón con solución salina hipertónica al 7.5\% más hidroxietilalmidón 200/0.5-6\%. La dosis máxima recomendada es de $250 \mathrm{ml}$ ó igual ó inferior a $5 \mathrm{ml} / \mathrm{kg}$ de peso en pacientes de peso inferior a $50 \mathrm{~kg}$.

Esta dosis es equivalente en efecto volémico a 2000cc de cristaloides y su efecto dura 1 hora. Se ha cuestionado el riesgo de aumento/producción de hematomas en cavidades cerradas afectadas por traumatismos, que deberá equilibrarse con los posibles beneficios de esta terapia de restitución volémica en pacientes en choque hemorrágico grave prehospitalario. No parece interferir con la hemostasia sí no se sobrepasa la dosis límite recomendada $(5 \mathrm{ml} / \mathrm{kg}$ de peso). Estos hechos harían recomendable esta sustancia en el tratamiento de los pacientes objetos de investigación (Rodríguez, Echevarría, \& Díaz, 2015), (Perel, Roberts, \& Ker, 2013), (Perel, Roberts, \& Ker, 2013).

Lo anterior planteado muestra la ventaja teórico-práctico de esta sustancia sobre el cloro sodio al 0,9\%, como lo demuestra el presente estudio, debido a que con dosis mucho menor garantiza mayor expansión de volumen y respuestas más duraderas.

Los eventos adversos en la presente investigación se comportaron igual que la literatura revisada (Rocha S. , 2016), (Hundley, Brooks, Thomovsky, \& Johnson, 2016); ambas sustancias tienen cierta similitud al plasma humano, aunque en mayor concentración en sales, por lo que los eventos adversos que suelen presentarse son ligeros, no obstante, la necesidad de dosis más altas con el cloro sodio al 0,9\% y menor capacidad de expandir el volumen, hacen que aparezcan más complicaciones, como ocurrió en la presente investigación.

\section{Conclusiones:}

El análisis muestra que de los pacientes que completaron el período de tratamiento con el cloro sodio hipertónico $(7,5 \%)$ y el control, mostró que el primero es más efectivo en el tratamiento del paciente con choque hemorrágico traumático.

Ambas sustancias resultaron ser seguras por recogerse un número mínimo de efectos adversos a las dosis y según el esquema de administración aplicados en el estudio, aunque la sustancia experimental fue más segura.

\section{Referencias.}

Bouglé, A., Harrois, A., \& Duranteau, J. (2012). Resuscitative strategies in traumatic hemorrhagic shock. Annals of Intensive Care, 3(1). doi:10.1186/2110-5820-3-1 
Rev. SINAPSIS, Edición № 11, Vol. 2, Diciembre 2017

Burton, W., Britton, A., \& Todd, B. (Mayo de 2014). Arterial vs venous blood gas differences during hemorrhagic shock. World J Crit Care Med, 3(2), 55-60. doi:10.5492/wjccm.v3.i2.55

Cecconi, M., De Backer, D., Antonelli, M., \& Beale, R. (Diciembre de 2014). Consensus on circulatory shock and hemodynamic monitoring. Task force of the European Society of Intensive Care Medicine. Intensive Care Medicine, 40(12). doi:10.1007/s00134-014-3525-z

Chang, R., John, B., \& Holcomb, J. (2017). Optimal Fluid Therapy for Traumatic Hemorrhagic Shock. Crit Care Clin, 15-36. doi:http://dx.doi.org/10.1016/j.ccc.2016.08.007

Egea, J., Freire, M., \& Serrano, A. (Noviembre de 2014). Objetivos y nuevas estrategias de resucitación en el paciente traumatizado grave. Med Intensiva, 38(8). doi:10.1016/j.medin.2014.06.003

Gómez, V., Ayuso, F., \& Jiménez, G. (2008). Recomendaciones de buena práctica clínica: atención inicial al paciente politraumatizado. SEMERGEN - Medicina de Familia, 354-363. doi:https://doi.org/10.1016/S1138-3593(08)72338-4

Guerrero, G., \& Andrighetti, L. (2011). Reposición de volumen en el politraumatizado. REV. MED. CLIN. CONDES, 22(5), 599-606. doi:http://dx.doi.org/10.1016/S0716-8640(11)70471-0

Hundley, D., Brooks, A., Thomovsky, E., \& Johnson, P. (2016). Crystalloids: A Quick Reference for Challenges in Daily Practice. Topics in Companion Animal Medicine, 31(2), 46-53. doi:https://doi.org/10.1053/j.tcam.2016.08.004

Jamal, S., Mandel, Y., \& Barnea, O. (2014). Optimization of Oxygen Delivery in Fluid Resuscitation for Hemorrhagic Shock: A Computer Simulation Study. Cardiovascular Engineering and Technology, 5(1), 82-95. doi:10.1007/s13239-013-0169-z

Kapp, M. (2005). The legal status of clinical practice parameters: an annotated bibliography. Am J Med Qual, 8(1). Obtenido de https://scholar.google.es/scholar?hl=es\&q=The+legal+status+of+clinical+practice+parameters $\% 3 \mathrm{~A}+\mathrm{an}+$ annotated+bibliography.+Am+J+Med+Qual\&btnG=\&lr

Navarro, S., Koo, M., Orrego, C., \& Muñoz, J. (2014). Estudio para la mejoría de la atención hospitalaria inicial del paciente politraumatizado: proyecto TRAUMACAT. Medicina Clínica, 143(1), 25-31. doi:https://doi.org/10.1016/j.medcli.2013.07.001

Perel, P., Roberts, I., \& Ker, K. (2013). Colloids versus crystalloids for fluid resuscitation in critically ill patients. Cochrane Database of Systematic Reviews. doi:10.1002/14651858.CD000567.pub6.

Perel, P., Roberts, I., \& Ker, K. (2013). Schierhout Colloids versus crystalloids for fluid resuscitation in critically ill patients (Review). Cochrane Database of Systematic Reviews.

Pinsky, M. (June de 2014). Functional Hemodynamic Monitoring: Current Concepts in Critical Care Published in final edited form as: Curr Opin Crit Care. 3, 288-293. doi:doi:10.1097/MCC.0

Raghunathan, K., Murray, P., Beattie, W., Lobo, D., Myburgh, J., \& Sladen, R. (2014). Choice of fluid in acute illness: what should be given? Aninternational consensus British Journal of Anaesthesia. Br J Anaesth, 13(5). doi:doi:10.1093/bja/aeu301 
Rev. SINAPSIS, Edición № 11, Vol. 2, Diciembre 2017

Rocha, S. (2016). Cardiovascular effects of shock and trauma in experimental models. A review. Braz J Cardiovasc Surg, 31(1), 45-51. Obtenido de https://scholar.google.es/scholar?hl=es\&q=Cardiovascular+Effects+of+Shock+and+Trauma+in +Experimental+Models.+A+Review\&btnG=\&lr=lang_es\%7Clang_

Rocha, S., \& Poli de Figueiredo, D. (2014). Hypertonic saline for treatment of shock: have welooked for everything? MEDICALEXPRESS. Obtenido de http://www.scielo.br/scielo.php?pid=S235804292014000100014\&script=sci_abstract\&tlng=pt $17-$

Rodríguez, T., Echevarría, A., \& Díaz, C. (2015). Criterios actuales de la reposición de volumen en pacientes con traumas. Revista Cubana de Anestesiología y Reanimación, 14(2). Obtenido de http://scielo.sld.cu/scielo.php?script=sci_arttext\&pid=S1726-67182015000200008

Rossaint, R., Bouillon, B., Cerny, V., \& Coats, T. (2016). The European guideline on management of major bleeding and coagulopathy following trauma. Fourth edition Rossaint et al. Critical Care, 20(100). doi:DOI 10.1186/s13054-016-1265

Scalea, T., Maltz, S., \& Yelon, J. (2014). Resuscitation of multiple trauma and head injury: role of crystalloid fluids and inotropes. Med Intensiva, 38(8). doi:10.1016/j.medin.2014.06.003

Scheeren, W., Wiesenack, C., Gerlach, H., \& Gernot, M. (2013). Goal-directed intraoperative fluid therapy guided by strokevolume and its variation in high-risk surgical patients: a prospective randomized multicentre study. J Clin Monit Comput, 27, 225-233. doi:DOI 10.1007/s10877013-9461-6

Schreiber, M., Meier, E., Tisherman, S., Kerby, J., Newgard, C., \& Brasel, K. (2015). A controlled resuscitation strategy is feasible and safe in hypotensive trauma patients: results of a prospective randomized pilot trial. J Trauma Acute Care Surg, 687-697.

doi:doi:10.1097/TA.0000000000000600.

Serracant, B., Montmany, S., Llaquet, B., Rebasa, C., Campos, S., \& Navarro, S. (2016). Registro prospectivo de politraumatismos graves. Ana' lisis de 1.200 pacientes. CIRUGIA ESPAÑOLA, $16-21$.

Shoemaker, W., Appel, P., \& Kram, H. (1993). Hemodynamic and oxygen transport responses in survivors and nonsurvivors of high risk surgery. Crit Care Med, 21, 977-990. Obtenido de https://scholar.google.es/scholar?hl=es\&q=16.\%09Shoemaker+WC\%2C+Appel+PL\%2C+Kra $\mathrm{m}+\mathrm{HB} \% 3 \mathrm{~A}+$ Hemodynamic+and+oxygen+transport+responses+in

Shoemaker, W., Appel, P., \& Kram, H. (1999). Hemodynamic and oxygen transport responses in survivors and nonsurvivors of high risk surgery. Crit Care Med. Obtenido de https://scholar.google.es/scholar?hl=es\&q=Hemodynamic+and+oxygen+transport+responses $+\mathrm{i}$ $\mathrm{n}+$ survivors+and+nonsurvivors+of+high+risk+surgery $\& b \operatorname{tnG}=\&$

Sigüenza, G., \& Vicuña, P. (2015). Evaluación del Protocolo de Atención Prehospitalaria para emergencias médicas en politraumatismos atendidos por el Cuerpo de Bomberos y Sistema ECU 911. Tesis de pregrado de Enfermeria. Azogues: UNIVERSIDAD DE CUENCA 
Rev. SINAPSIS, Edición № 11, Vol. 2, Diciembre 2017

FACULTAD DE CIENCIAS MÉDICAS ESCUELA DE ENFERMERÍA. Obtenido de http://dspace.ucuenca.edu.ec/handle/123456789/23065

\section{Anexos}

Tabla 1. Distribución de pacientes por grupos según la localización anatómica de las lesiones y etiología.

\begin{tabular}{|l|l|l|l|l|l|l|}
\hline \multirow{2}{*}{$\begin{array}{l}\text { Localización Anatómica y } \\
\text { Etiología }\end{array}$} & \multicolumn{3}{l|}{ Experimental } & \multicolumn{2}{l|}{ Control } & \multicolumn{2}{l|}{ Significación } \\
\cline { 2 - 8 } & No & $\%$ & No & $\%$ & No & P \\
\hline Localización Anatómica & & & & & & \\
\hline Cráneo Facial & 6 & 18,7 & 7 & 21,9 & 13 & 0,666 \\
\hline Tórax & 7 & 21,9 & 5 & 15,6 & 12 & 0,525 \\
\hline Abdomen & 15 & 46,9 & 17 & 53,1 & 32 & 0,619 \\
\hline Extremidades & 4 & 12,5 & 3 & 9,4 & 7 & 0,691 \\
\hline Total & 32 & 100 & 32 & 100 & 64 & \\
\hline Según etiología & & & & & & \\
\hline Arma blanca & 14 & 43,7 & 11 & 34,4 & 25 & 0,545 \\
\hline Accidentes del tránsito & 12 & 37,5 & 13 & 40,6 & 25 & 0,799 \\
\hline Caídas de alturas & 4 & 12,5 & 5 & 15,6 & 9 & 0,711 \\
\hline Otros & 2 & 6,3 & 3 & 9,4 & 5 & 0,643 \\
\hline Total & 32 & 100 & 32 & 100 & 64 & \\
\hline
\end{tabular}

Tabla 2. Comportamiento de la normalidad de la TAM y frecuencia cardiaca en cada uno de los momentos de realizadas las mediciones.

\begin{tabular}{|l|l|l|l|l|l|l|}
\hline \multirow{2}{*}{$\begin{array}{l}\text { Momento de } \\
\text { realizadas las } \\
\text { mediciones. }\end{array}$} & \multicolumn{4}{l|}{ Kolmogorov-Smirnov } \\
\cline { 2 - 7 } & $\begin{array}{l}\text { Estadístic } \\
\text { o g1 }\end{array}$ & Sig. & $\begin{array}{l}\text { Estadísti } \\
\text { co }\end{array}$ & gl & Sig. \\
\hline $\begin{array}{l}\text { Normalidad de } \\
\text { TAM }\end{array}$ & & & & & & \\
\hline TAM P &, 090 & 64 &, 200 &, 949 & 64 &, 010 \\
\hline TAM 30 &, 157 & 64 &, 000 &, 828 & 64 &, 000 \\
\hline TAM 60 &, 153 & 64 &, 001 &, 886 & 64 &, 000 \\
\hline TAM 90 &, 219 & 64 &, 000 &, 762 & 64 &, 000 \\
\hline TAM 120 &, 269 & 64 &, 000 &, 716 & 64 &, 000 \\
\hline
\end{tabular}


Rev. SINAPSIS, Edición № 11, Vol. 2, Diciembre 2017

\begin{tabular}{|l|l|l|l|l|l|l|}
\hline TAM 24 &, 253 & 64 &, 000 &, 614 & 64 &, 000 \\
\hline TAM ALTA &, 319 & 64 &, 000 &, 574 & 64 &, 000 \\
\hline $\begin{array}{l}\text { Normalidad de } \\
\text { la Frecuencia. }\end{array}$ &, 132 & 64 &, 008 &, 889 & 64 &, 000 \\
\hline FC &, 201 & 64 &, 000 &, 829 & 64 &, 000 \\
\hline \multicolumn{1}{|c|}{ FC30 } &, 210 & 64 &, 000 &, 846 & 64 &, 000 \\
\hline FC60 &, 193 & 64 &, 000 &, 856 & 64 &, 000 \\
\hline FC90 &, 181 & 64 &, 000 &, 802 & 64 &, 000 \\
\hline FC120 &, 271 & 64 &, 000 &, 686 & 64 &, 000 \\
\hline FC24 &, 286 & 64 &, 000 &, 633 & 64 &, 000 \\
\hline FCALTA & & & & & & \\
\hline & & & & & & \\
\hline
\end{tabular}

Tabla 3: Comportamiento de la HTA-Media Pre- durante el tratamiento y Diuresis en ambos grupos a. Diferencia de medias para muestras independientes para la HTA-M al inicio

\begin{tabular}{|l|l|l|l|}
\hline Momento de realizada la medición & Grupo Experimental & Grupo Control & $\begin{array}{l}\text { Probabilida } \\
\text { d }\end{array}$ \\
\cline { 2 - 4 } Comportamiento de la TAM. & & & \\
\hline TAM pretratamiento & $\begin{array}{l}49,3333 \text { (Media) } \\
11,6098 \text { (Desviación } \\
\text { típica) }\end{array}$ & $\begin{array}{l}51,4375 \text { (Media) } \\
12,4858 \text { (Desviación } \\
\text { típica) }\end{array}$ & 0,4877 \\
\hline $\begin{array}{l}\text { Comportamiento de la HTA en } \\
\text { cada }\end{array}$ & & & \\
\hline TAM30 & 61,6771 & 62,3021 & 0,115 \\
\hline TAM60 & 67,9062 & 72,8750 & 0,010 \\
\hline TAM90 & 72,7396 & 77,3646 & 0,019 \\
\hline TAM120 & 74,6875 & 79,6250 & 0,002 \\
\hline TAM24 & 83,0625 & 81,6875 & 0,791 \\
\hline TAMALTA & 88,7500 & 83,2292 & 0,151 \\
\hline Comportamiento de la diuresis * & & & 0,476 \\
\hline DIUR120 & 79,25 & 87,72 & 0,012 \\
\hline DIUR24HO & 2324,31 & 1456,56 & \\
\hline
\end{tabular}


Rev. SINAPSIS, Edición № 11, Vol. 2, Diciembre 2017

ISSN 1390 - 9770

* Comportamiento de la HTA y diuresis en cada uno de los momentos de realizadas las mediciones.

Prueba de Mann-Whitney.

Tabla 4 Comportamiento de la normalidad de saturación y diuresis en cada uno de los momentos de realizadas las mediciones.

\begin{tabular}{|c|c|c|c|c|c|c|}
\hline \multirow{2}{*}{$\begin{array}{l}\text { Momento de } \\
\text { realizadas las } \\
\text { mediciones. }\end{array}$} & \multicolumn{3}{|c|}{ Kolmogorov-Smirnov } & \multicolumn{3}{|c|}{ Shapiro-Wilk } \\
\hline & $\begin{array}{l}\text { Estadístic } \\
\mathrm{O}\end{array}$ & $\mathrm{gl}$ & Sig. & $\begin{array}{l}\text { Estadístic } \\
\mathrm{o}\end{array}$ & gl & Sig. \\
\hline \multicolumn{7}{|c|}{$\begin{array}{l}\text { Comportamient } \\
\text { o de saturación } \\
\text { de }\end{array}$} \\
\hline SPO2 & ,263 & 64 &, 000 & ,851 & 64 & ,000 \\
\hline SP30 &, 250 & 64 & ,000 & ,860 & 64 & ,000 \\
\hline SP60 & ,261 & 64 & ,000 & ,902 & 64 &, 000 \\
\hline SP90 & 409 & 64 & 000 &, 254 & 64 &, 000 \\
\hline SP120 & ,416 & 64 & ,000 & ,253 & 64 & ,000 \\
\hline SP24HORA & ,414 & 64 &, 000 &, 255 & 64 &, 000 \\
\hline SPALTA & ,489 & 64 &, 000 & ,347 & 64 &, 000 \\
\hline \multicolumn{7}{|c|}{$\begin{array}{l}\text { Normalización } \\
\text { de Diuresis }\end{array}$} \\
\hline DIUR60 & ,091 & 64 & ,200(*) & ,975 & 64 & ,223 \\
\hline DIUR120 &, 130 & 64 & ,009 & ,874 & 64 &, 000 \\
\hline DIUR24HO & ,432 & 64 &, 000 & ,205 & 64 &, 000 \\
\hline
\end{tabular}

Tabla 5. Comportamiento de la frecuencia cardiaca y saturación de oxígeno en cada uno de los momentos de realizadas las mediciones. Prueba de Mann-Whitney.

\begin{tabular}{|l|l|l|l|}
\hline $\begin{array}{l}\text { Momento de realizadas } \\
\text { las mediciones. }\end{array}$ & & & \multirow{2}{*}{ Probabilidad } \\
\cline { 2 - 3 } & Grupo Experimental & Grupo Control & \\
\hline $\begin{array}{l}\text { Comportamiento de la } \\
\text { frecuencia cardiaca }\end{array}$ & & & \\
\hline FC PRE T & 125,56 & 132,31 & 0,031 \\
\hline FC30 & 124,59 & 134,15 & 0,000 \\
\hline FC60 & 112,90 & 137,12 & 0,000 \\
\hline
\end{tabular}


Rev. SINAPSIS, Edición № 11, Vol. 2, Diciembre 2017

\begin{tabular}{|c|c|c|c|}
\hline FC90 & 109,43 & 123,90 & 0,000 \\
\hline FC120 & 106,37 & 120,50 & 0,000 \\
\hline FC24 & 104,43 & 110,15 & 0,000 \\
\hline FCALTA & 74,56 & 82,65 & 0,000 \\
\hline \multicolumn{4}{|c|}{$\begin{array}{l}\text { Comportamiento de la } \\
\text { saturación }\end{array}$} \\
\hline $\mathrm{SPO}_{2} \mathrm{PT}$ & 87,47 & 91,90 & 0,000 \\
\hline $\mathrm{SPO}_{2} 30$ & 88,78 & 92,81 & 0,000 \\
\hline $\mathrm{SPO}_{2} 60$ & 94,06 & 93,81 & 0,000 \\
\hline $\mathrm{SPO}_{2} 90$ & 97,03 & 91,84 & 0,000 \\
\hline $\mathrm{SPO}_{2} 120$ & 97,34 & 92,09 & 0,000 \\
\hline $\mathrm{SPO}_{2} 24 \mathrm{H}$ & 97,94 & 92,56 & 0,000 \\
\hline $\mathrm{SPO}_{2} \mathrm{ALTA}$ & 93,34 & 87,72 & 0,000 \\
\hline
\end{tabular}

Tabla 6. Comportamiento de la diuresis en ambos grupos al inicio del tratamiento. Diferencia de medias para muestras independientes.

\begin{tabular}{|c|c|c|c|}
\hline \multirow{2}{*}{$\begin{array}{l}\text { Momento de } \\
\text { realizada la } \\
\text { medición }\end{array}$} & \multicolumn{2}{|l|}{ DIURESIS } & \multirow[t]{2}{*}{ Probabilidad } \\
\hline & $\begin{array}{l}\text { Grupo } \\
\text { Experimental }\end{array}$ & Grupo Control & \\
\hline $\begin{array}{l}\text { TAM } \\
\text { pretratamiento }\end{array}$ & $\begin{array}{l}\text { 41,2188 (Media) } \\
20,66591 \\
\text { (Desviación } \\
\text { típica) }\end{array}$ & $\begin{array}{l}\text { 28,6875 (Media) } \\
\text { 13,80144 } \\
\text { (Desviación típica) }\end{array}$ & 0,006 \\
\hline
\end{tabular}


Rev. SINAPSIS, Edición № 11, Vol. 2, Diciembre 2017

Gráfico 1. Distribución de pacientes por grupos según la ocurrencia de eventos adversos.

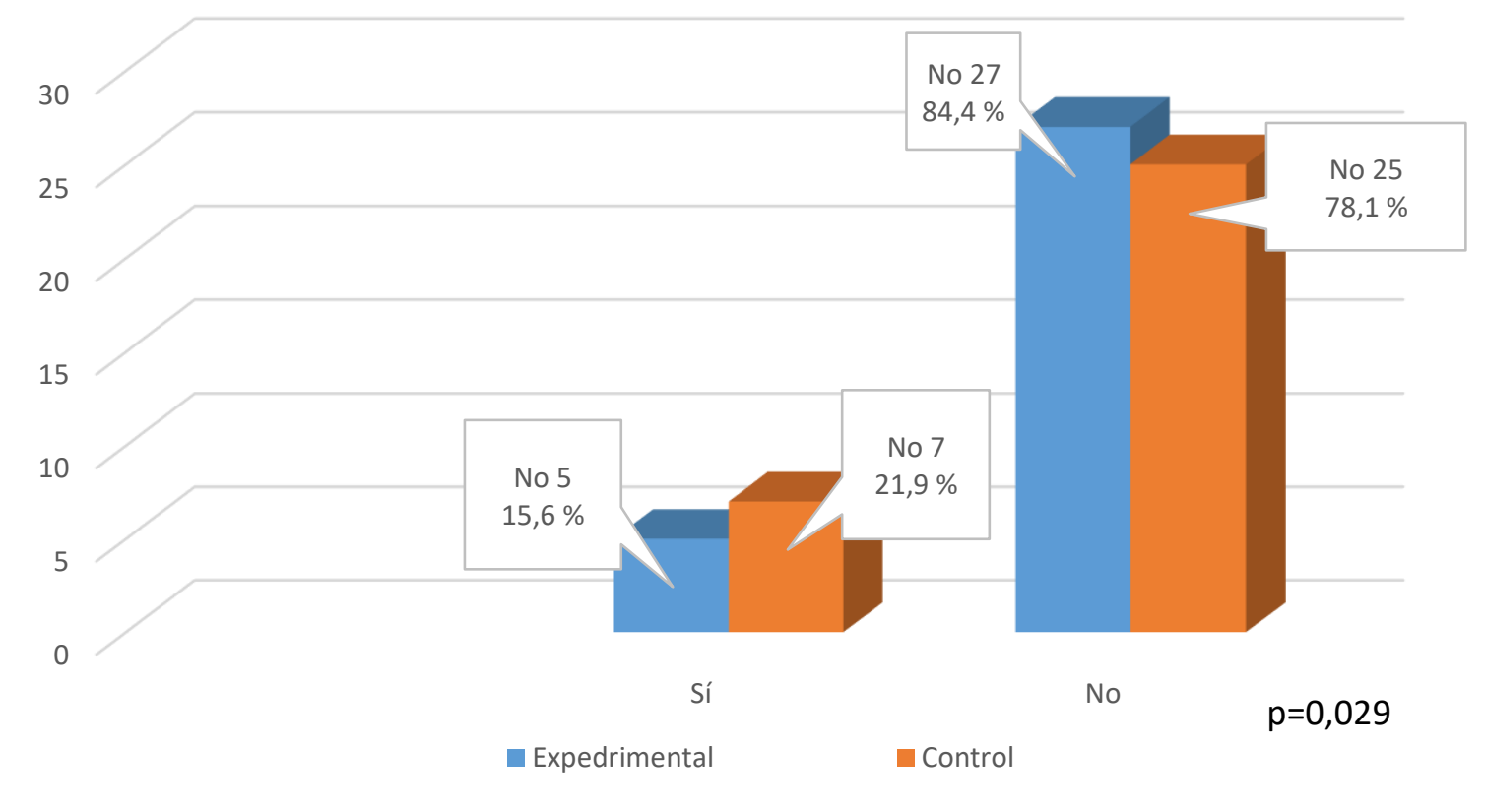

